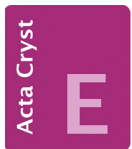

CRYSTALLOGRAPHIC COMMUNICATIONS

ISSN 2056-9890

\section{Crystal structure of a three-dimensional neodymium(III) coordination polymer, $\left[\mathrm{Nd}_{2}\left(\mathrm{H}_{2} \mathrm{O}\right)_{6}\right.$ (glutarato $\left.)\left(\mathrm{SO}_{4}\right)_{2}\right]_{n}$}

\author{
Saranphong Yimklan, ${ }^{\mathrm{a} *}$ Yothin Chimupala, ${ }^{\mathrm{b}}$ Sutsiri Wongngam ${ }^{\mathrm{a}}$ and Nippich \\ Kaeosamut $^{a}$
}

Received 14 September 2021

Accepted 5 January 2022

Edited by $\mathrm{V}$. Jancik, Universidad Nacional Autónoma de México, México

Keywords: crystal structure; coordination polymer; lanthanide; glutarate.

CCDC reference: 2107848

Supporting information: this article has supporting information at journals.iucr.org/e

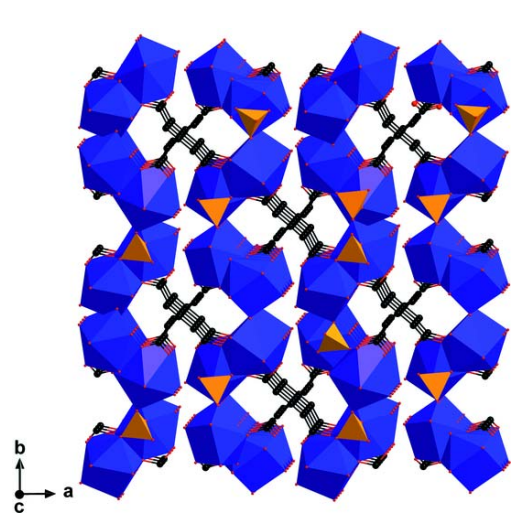

\section{Structural commentary}

The coordination network $\mathbf{1},\left[\mathrm{Nd}_{2}\left(\mathrm{H}_{2} \mathrm{O}\right)_{6} \text { (glutarato) }\left(\mathrm{SO}_{4}\right)_{2}\right]_{n}$ crystallizes in the monoclinic $P 2_{1} / c$ space group. There are two crystallographically independent $\mathrm{Nd}^{\mathrm{III}}$ cations ( $\mathrm{Nd} 1$ and $\left.\mathrm{Nd} 2\right)$, 
two sulfate anions, and six coordinated water molecules in the asymmetric unit, as illustrated in Fig. 1.

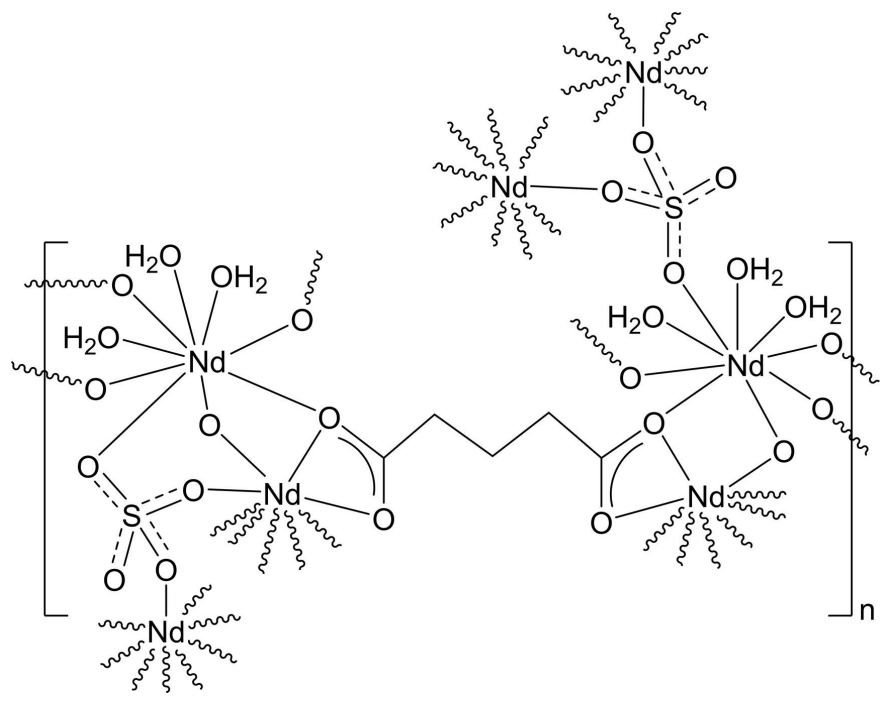

Both $\mathrm{Nd}^{\mathrm{III}}$ cations are nine-coordinated to $\mathrm{O}$ atoms from one bridging glutarate ${ }^{2-}$, two chelating glutarate ${ }^{2-}$, two chelating sulfate anions and three coordinated $\mathrm{H}_{2} \mathrm{O}$, adopting a distorted tricapped trigonal-prismatic geometry, TPRS$\left\{\mathrm{Nd}^{\mathrm{III}} \mathrm{O}_{9}\right\}$ (see Fig. $1 b$ ), forming an edge-sharing dinuclear unit with its symmetry-related $\mathrm{Nd}^{\mathrm{III}} \mathrm{O}_{9}$ polyhedron. The $\mathrm{Nd}^{\mathrm{III}}-\mathrm{O}$ bond distances are in the range of 2.383 (2)-2.785 (2) $\AA$, which are reasonable and comparable to those reported for other $\mathrm{Nd}^{\mathrm{III}}$ coordination polymers such as $\left[\mathrm{Nd}\left(\mathrm{H}_{2} \mathrm{O}\right)_{4}\right.$ (glutarato $\left.)\right] \mathrm{Cl}$ (Hussain et al., 2015), $\left[\mathrm{Nd}\left(\mathrm{H}_{2} \mathrm{O}\right)_{4}\right.$ (glutarato) $] \mathrm{Cl} \cdot 2 \mathrm{H}_{2} \mathrm{O}$ (Legendziewicz et al., 1999) and $\left[\mathrm{Nd}_{2}\left(\mathrm{H}_{2} \mathrm{O}\right)_{2}\right.$ (glutarato) $] \cdot 2 \mathrm{H}_{2} \mathrm{O}$ (Głowiak et al., 1986). In contrast to the above-mentioned coordination polymers, $\left[\mathrm{Nd}\right.$ (glutarato) $\left.\left(\mathrm{H}_{2} \mathrm{O}\right)_{4}\right] \mathrm{Cl}$ (Hussain et al., 2015) and $\left[\mathrm{Nd}\right.$ (glutarato) $\left.\left(\mathrm{H}_{2} \mathrm{O}\right)_{4}\right] \mathrm{Cl} \cdot 2 \mathrm{H}_{2} \mathrm{O}$ (Legendziewicz et al., 1999) consisting of cationic $\left\{\mathrm{Nd}\left(\mathrm{H}_{2} \mathrm{O}\right)_{\mathrm{x}}(\text { glutarato })\right\}_{n}^{n+}$ $(x=2,4)$ subunits compensated by uncoordinated chloride anions, each of the tetrahedral $\mathrm{SO}_{4}{ }^{2-}$ ligands in $\mathbf{1}$ links three adjacent $\mathrm{Nd}^{\mathrm{III}}$ atoms, forming a neutral two-dimensional network of $\left[\mathrm{Nd}_{2}\left(\mathrm{H}_{2} \mathrm{O}\right)_{6}\right.$ (glutarato $\left.)\left(\mathrm{SO}_{4}\right)_{2}\right]_{n}$. The $\mathrm{S}-\mathrm{O}$ bond distances are in the range 1.449 (3)-1.485 (2) $\AA$, with $\mathrm{O}-\mathrm{S}-$ $\mathrm{O}$ angles ranging from $107.78(16)$ to $111.67(15)^{\circ}$. The flexible glutarate linker exhibits a $\left(\mu_{4}-\kappa^{2} O: \kappa \mathrm{O}^{\prime}: \kappa^{2} \mathrm{O}^{\prime \prime}: \kappa O^{\prime \prime \prime}\right.$ coordination mode with an anti-anti conformation as depicted in Fig. $2 a$. There are six crystallographically independent water (a)

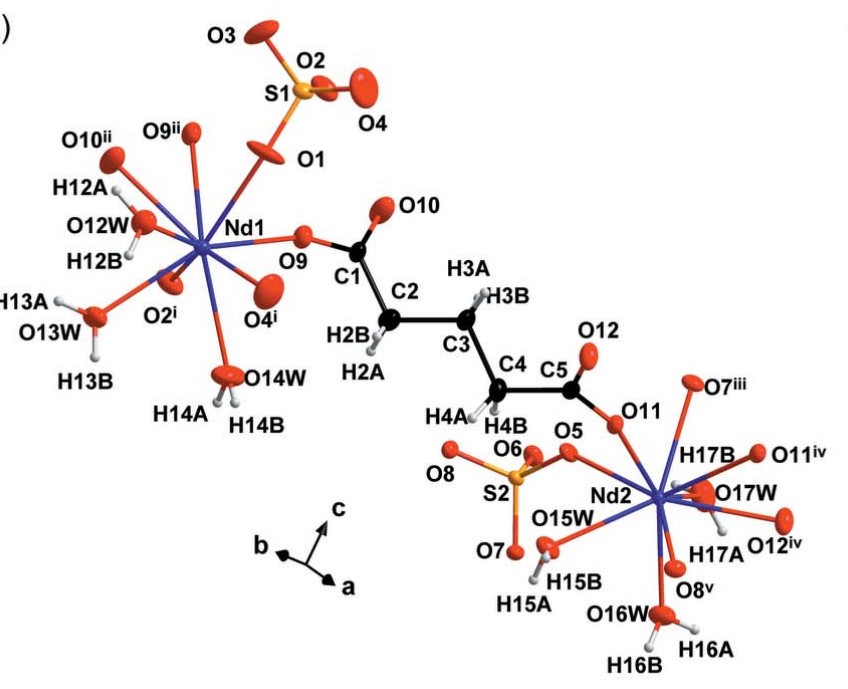

(b)
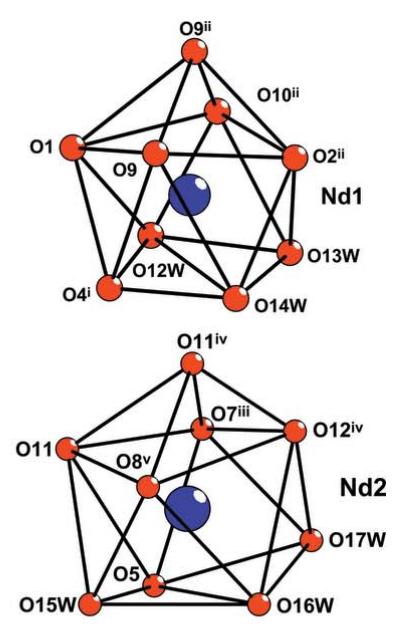

(c)

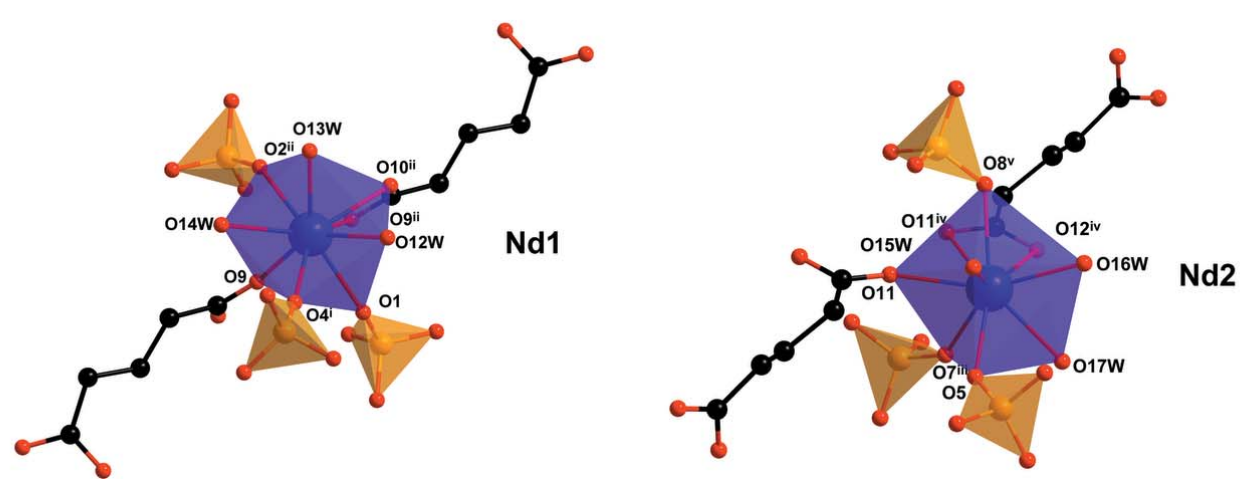

Figure 1

Graphical representations of $(a)$ an extended asymmetric unit of $\mathbf{1}$ drawn with $50 \%$ probability ellipsoids, $(b)$ coordination geometries of Nd1 (top) and $\mathrm{Nd} 2$ (bottom) and (c) coordination environment of Nd1 (left) and Nd2 (right). [Symmetry codes: (i) $-x+2,-y,-z ;$ (ii) $-x+2, y-1 / 2,-z-\frac{1}{2}$; (iii) $x$, $-y+\frac{1}{2}, z+\frac{1}{2} ;$ (iv) $-x+1,-y+1,-z ;$ (v) $x,-y+\frac{1}{2}, z-\frac{1}{2}$; (vi) $-x+2, y+\frac{1}{2},-z-\frac{1}{2}$.] 
molecules completing the coordination sites of the two $\mathrm{Nd}^{\mathrm{III}}$ atoms (three $\mathrm{H}_{2} \mathrm{O}$ molecules for each $\mathrm{Nd}^{\mathrm{III}}$ atom, Fig. $2 b$ ).

\section{Supramolecular features}

The polymeric structure of $\mathbf{1}$ can be described as a threedimensional non-porous framework, which is constructed from edge-sharing TPRS- $\left\{\mathrm{Nd}^{\mathrm{III}} \mathrm{O}_{9}\right\}$ polyhedra linked through sulfate anions, acting as tritopic inorganic linkers, into a cationic $\left[\mathrm{Nd}_{2}\left(\mathrm{H}_{2} \mathrm{O}\right)_{6}\left(\mathrm{SO}_{4}\right)_{2}\right]_{n}{ }^{2 n+}$ sheets parallel to the $(011)$ layers, as illustrated in Fig. $3 a$. It is noteworthy that these sheets also contain large inorganic $\left[\mathrm{Nd}\left(\mathrm{SO}_{4}\right)\right]_{4}$ rings further stabilized by $\mathrm{O}-\mathrm{H} \cdots \mathrm{O}$ hydrogen bonds between the water molecules and sulfate anions (Table 1). Eventually, the final three-dimensional network is formed by connecting these adjacent cationic sheets by the glutarate ligands (Fig. 3b). This three-dimensional arrangement also features $\mathrm{O}-\mathrm{H} \cdots \mathrm{O}$ hydrogen bonds between two water molecules or between a water molecule and oxygen atoms of the glutarate ligands (Fig. 2b). In total, all but one hydrogen atom from the six crystallographically independent water molecules are involved in hydrogen bonding (Table 1). Analysis of these hydrogen bonds revealed thirteen different first-order graph sets (Bernstein et al., 1995) consisting of five rings and eight different chains.

\section{Database survey}

A search of the Cambridge Structural Database (CSD, Version 5.42, update of September 2021; Groom et al., 2016) confirms that no $\mathrm{Nd}^{\mathrm{III}}$ coordination polymer containing both glutarate ${ }^{2-}$ and $\mathrm{SO}_{4}{ }^{2-}$ has been reported. However, several

(a)

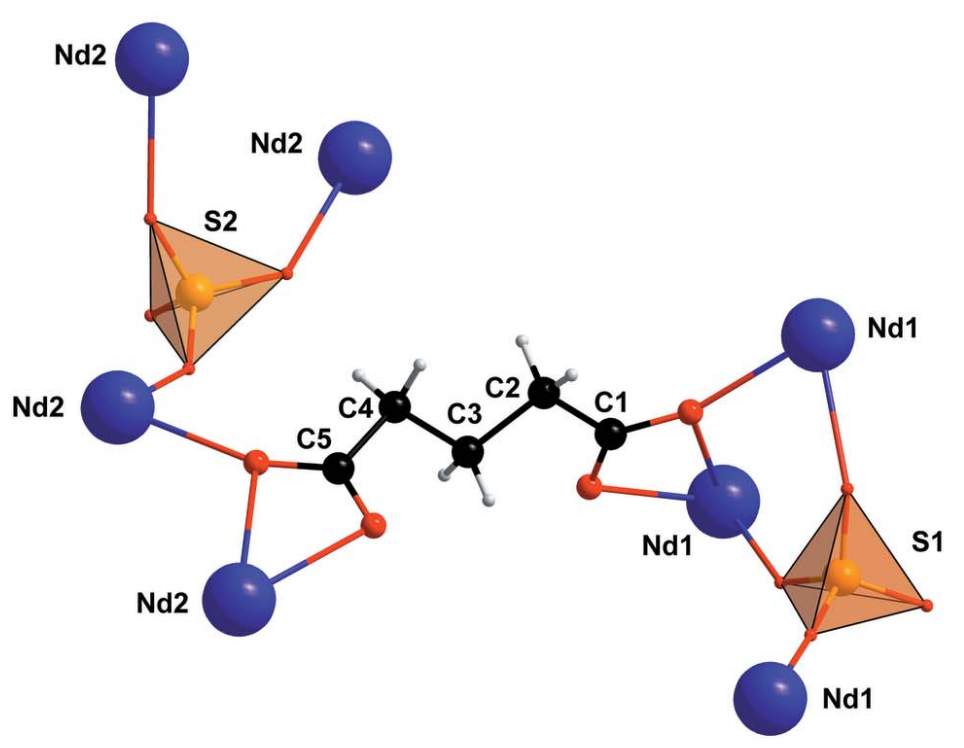

(b)

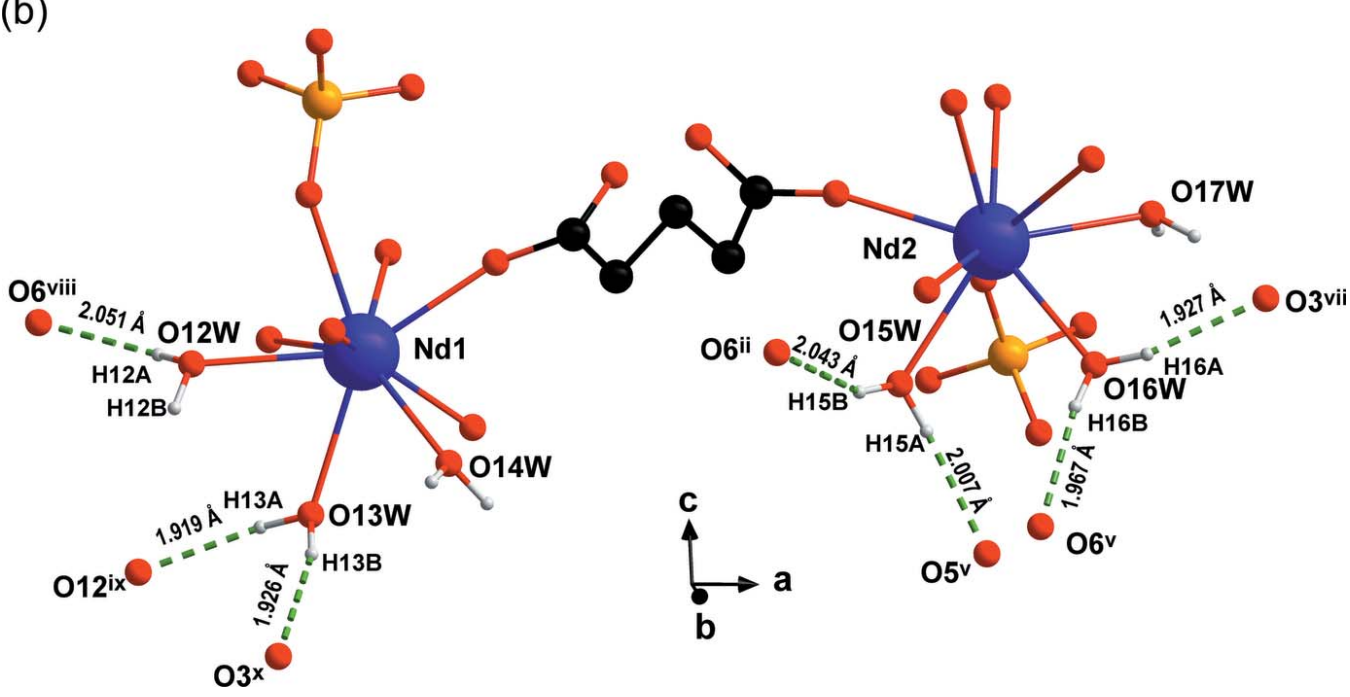

Figure 2

Depictions of $(a)$ the coordination modes of the glutarate and the sulfate ligands in $\mathbf{1}$ and $(b)$ seven of the eleven crystallographically independent hydrogen bonds (dashed green lines) with bond distances. [Symmetry codes: (i) $-x+2,-y,-z$; (ii) $-x+2, y-\frac{1}{2},-z-\frac{1}{2}$; (iii) $x,-y+\frac{1}{2}, z+\frac{1}{2}$; (iv) $-x+1$, $-y+1,-z$; (v) $x,-y+\frac{1}{2}, z-\frac{1}{2}$; (vi) $-x+2, y+\frac{1}{2},-z-\frac{1}{2}$; (vii) $x+1,-y+\frac{1}{2}, z-\frac{1}{2}$; (viii) $x-1, y, z$; (ix) $-x+1, y+\frac{1}{2},-z-\frac{1}{2}$; (x) $x, y, z-1$.] 
Table 1

Hydrogen-bond geometry $\left(\AA,^{\circ}\right)$.

\begin{tabular}{|c|c|c|c|c|}
\hline$D-\mathrm{H} \cdots A$ & $D-\mathrm{H}$ & $\mathrm{H} \cdots A$ & $D \cdots A$ & $D-\mathrm{H} \cdots A$ \\
\hline $\mathrm{O} 16 W-\mathrm{H} 16 A \cdots \mathrm{O} 3^{\mathrm{i}}$ & 0.85 & 1.91 & $2.709(4)$ & 155 \\
\hline $\mathrm{O} 16 W-\mathrm{H} 16 B \cdots \mathrm{O} 6^{\mathrm{ii}}$ & 0.85 & 1.94 & $2.774(3)$ & 165 \\
\hline $\mathrm{O} 17 W-\mathrm{H} 17 A \cdots \mathrm{O} 12 W^{\mathrm{iii}}$ & $0.81(2)$ & $2.38(4)$ & $2.966(4)$ & $130(4)$ \\
\hline $\mathrm{O} 17 W-\mathrm{H} 17 B \cdots \mathrm{O} 3^{\mathrm{i}}$ & $0.84(2)$ & $2.09(2)$ & $2.903(4)$ & $165(4)$ \\
\hline $\mathrm{O} 12 W-\mathrm{H} 12 A \cdots \mathrm{O} 6^{\mathrm{iv}}$ & 0.85 & 2.10 & $2.825(4)$ & 143 \\
\hline $\mathrm{O} 12 W-\mathrm{H} 12 B \cdots \mathrm{O} 17 W^{\mathrm{v}}$ & 0.85 & 2.07 & $2.904(4)$ & 166 \\
\hline $\mathrm{O} 13 W-\mathrm{H} 13 A \cdots \mathrm{O} 12^{\mathrm{vi}}$ & 0.85 & 1.95 & $2.733(3)$ & 153 \\
\hline $\mathrm{O} 13 W-\mathrm{H} 13 B \cdots \mathrm{O}^{\mathrm{vii}}$ & 0.85 & 1.99 & $2.745(4)$ & 148 \\
\hline $\mathrm{O} 14 W-\mathrm{H} 14 A \cdots \mathrm{O} 13 W^{\mathrm{viii}}$ & $0.85(2)$ & $2.13(2)$ & $2.966(4)$ & $165(4)$ \\
\hline $\mathrm{O} 18-\mathrm{H} 18 A \cdots \mathrm{O} 6^{\mathrm{ix}}$ & $0.83(2)$ & $2.03(2)$ & $2.826(3)$ & $160(3)$ \\
\hline $\mathrm{O} 18-\mathrm{H} 18 B \cdots \mathrm{O} 5^{\mathrm{ii}}$ & $0.81(2)$ & $2.00(2)$ & $2.805(3)$ & $170(4)$ \\
\hline
\end{tabular}

Symmetry codes: (i) $x+1,-y+\frac{1}{2}, z-\frac{1}{2}$; (ii) $x,-y+\frac{1}{2}, z-\frac{1}{2}$; (iii) $x+1, y, z$; (iv) $x-1, y, z$; (v) $x-1,-y+\frac{1}{2}, z-\frac{1}{2}$; (vi) $-x+1, y+\frac{1}{2},-z-\frac{1}{2}$; (vii) $x, y, z-1$; (viii) $-x+1,-y+1,-z-1$; (ix) $-x+2, y-\frac{1}{2},-z-\frac{1}{2}$.

related polymeric structures, viz. catena- $[(\mu$-pentanedioato)tetraaquaneodymium chloride] (NEMXIP; Hussain et al., 2015), catena- $\left[\left(\mu_{4}\right.\right.$-glutarato $)$ tetraaquadineodymium chloride dihydrate] (DIQZAE01; Marsh, 2005), catena-[bis $\left(\mu_{4}\right.$-pentane-1,5-dionato) $\left(\mu_{2}\right.$-pentane-1,5-dionato)diaquadineodymium(III) tetrahydrate] (FAQYUR; Legendziewicz et al., 1999) and catena- $\left[\operatorname{tris}\left(\mu_{3}\right.\right.$-glutarato- $\left.O, O, O^{\prime}, O^{\prime \prime}, O^{\prime \prime \prime}\right)$ diaquadineodymium(III) dihydrate] (FAFGAU; Głowiak et al., 1986), have been reported.

\section{Synthesis and crystallization}

Complex 1 was synthesized by dissolving $\mathrm{Nd}_{2}\left(\mathrm{SO}_{4}\right)_{3} \cdot 8 \mathrm{H}_{2} \mathrm{O}$ ( $1 \mathrm{mmol}, 0.721 \mathrm{~g})$, glutaric acid (1 mmol, $0.132 \mathrm{~g})$, and 4, $4^{\prime}-$ bipyridine ( $1 \mathrm{mmol}, 0.156 \mathrm{~g})$ in $40.0 \mathrm{~mL}$ of deionized water under ambient conditions. The solution was transferred into an open glass reactor and then irradiated by microwaves $(800 \mathrm{~W})$ for 10 minutes. The solution was let to cool to ambient temperature. Pale-purple block-shaped crystals crystallized from the solution within a few minutes. FT-IR (ATR Mode, $\mathrm{cm}^{-1}$ ) of 1: $v_{\text {stretch }}(\mathrm{O}-\mathrm{H}) 3364, v_{\text {stretch }}(\mathrm{C}-\mathrm{H})$ 2990, $v_{a s}\left(\mathrm{COO}^{-}\right) 1531, v_{s}\left(\mathrm{COO}^{-}\right) 1430, \delta(\mathrm{O}-\mathrm{H}) 1355, v_{s}(\mathrm{~S}-$ O) $1101, v_{s}(\mathrm{~S}-\mathrm{O}) 1077, v_{s}\left(\mathrm{SO}_{4}{ }^{2-}\right) 596$.

(a)

Figure 3

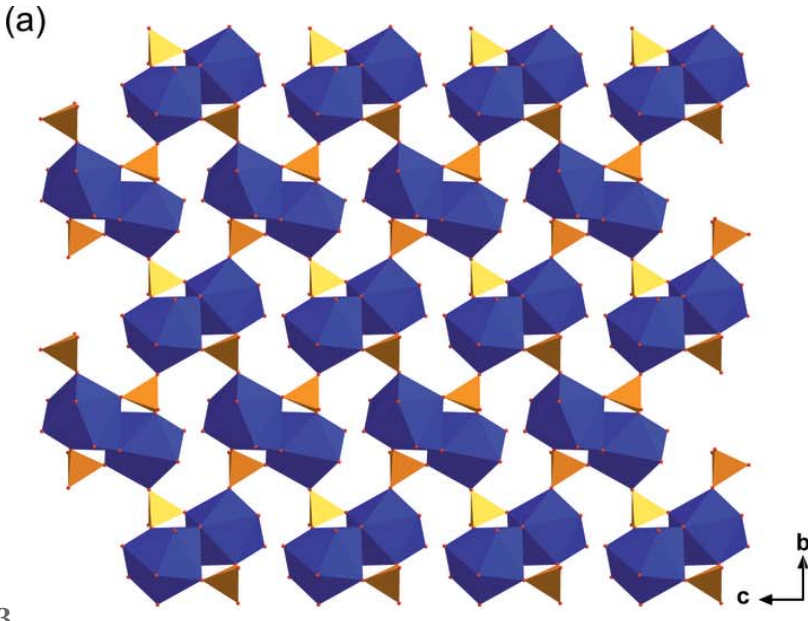

Table 2

Experimental details.

Crystal data

Chemical formula

$M_{\mathrm{r}}$

Crystal system, space group

Temperature (K)

$a, b, c(\AA)$

$\beta\left({ }^{\circ}\right)$

$V\left(\AA^{3}\right)$

$Z$

Radiation type

$\mu\left(\mathrm{mm}^{-1}\right)$

Crystal size (mm)

Data collection

Diffractometer

Absorption correction

$T_{\min }, T_{\max }$

No. of measured, independent and observed $[I>2 \sigma(I)]$ reflections

$R_{\text {in }}$

$(\sin \theta / \lambda)_{\max }\left(\AA^{-1}\right)$

Refinement

$R\left[F^{2}>2 \sigma\left(F^{2}\right)\right], w R\left(F^{2}\right), S$

No. of reflections

No. of parameters

No. of restraints

$\mathrm{H}$-atom treatment

$\Delta \rho_{\max }, \Delta \rho_{\min }\left(\mathrm{e} \AA^{-3}\right)$

Computer programs: CrysAlis PRO (Agilent, 2014), SHELXT2018/2 (Sheldrick, 2015a), SHELXL2018/3 (Sheldrick, 2015b) and OLEX2 (Dolomanov et al., 2009).

\section{Refinement}

Crystal data, data collection and structure refinement details are summarized in Table 2. Carbon-bound $\mathrm{H}$ atoms were positioned geometrically $(\mathrm{C}-\mathrm{H}=0.97 \AA)$ and constrained using the riding-model approximation with $U_{\text {iso }}(\mathrm{H})=$ $1.2 U_{\text {eq }}(\mathrm{C})$. The $\mathrm{H}$ atoms from the water molecules were located in the residual electron-density map, and where

Views of $(a)$ the $\left[\mathrm{Nd}_{2}\left(\mathrm{H}_{2} \mathrm{O}\right)_{6}\left(\mathrm{SO}_{4}\right)_{2}\right]_{n}^{2 n+}$ sheet and $(b)$ the three-dimensional framework of $\mathbf{1}$.

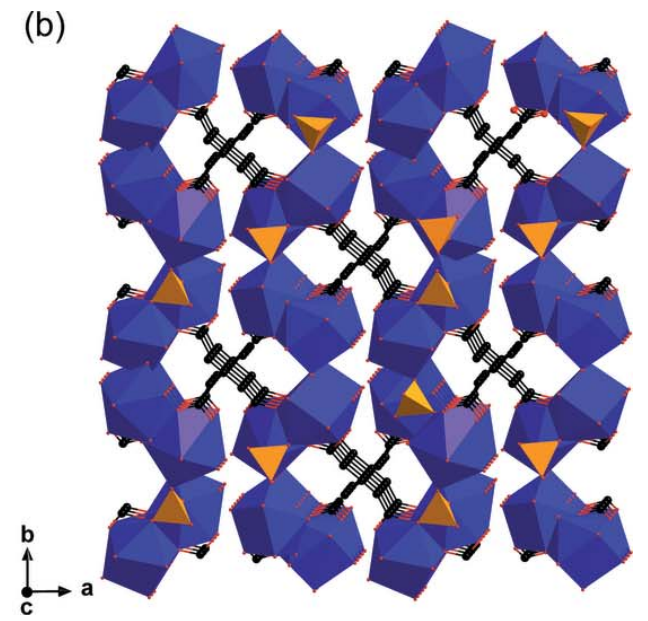


necessary, refined with distance and angle restraints or riding on the parent oxygen atom.

\section{Acknowledgements}

This research was partially supported by the CMU Junior Research Fellowship Program, Faculty of Science, Chiang Mai University, the Development and Promotion of Science and Technology Talent Project (DPST) through a research fund for graduates with first placement. The authors thank W. Booncharoen for chemical and physical analysis services during the COVID-19 pandemic. SY and YC acknowledge A. Rujiwatra for supervision in the DPST research fund. SW and NK also thank the DPST project for their research grants.

\section{Funding information}

Funding for this research was provided by: Faculty of Science, Chiang Mai University; Development and Promotion of Science and Technology Talent Project (DPST) through a research fund for graduates with first placement; CMU Junior Research Fellowship Program.

\section{References}

Agilent (2014). CrysAlis PRO. Agilent Technologies Ltd, Yarnton, England.

Bernstein, J., Davis, R. E., Shimoni, L. \& Chang, N.-L. (1995). Angew. Chem. Int. Ed. Engl. 34, 1555-1573.

Dolomanov, O. V., Bourhis, L. J., Gildea, R. J., Howard, J. A. K. \& Puschmann, H. (2009). J. Appl. Cryst. 42, 339-341.

Furukawa, H., Cordova, K. E., O'Keeffe, M. \& Yaghi, O. M. (2013). Science, 341, 1230444-1230444.

Głowiak, T., Dao-Cong Ngoan \& Legendziewicz, J. (1986). Acta Cryst. C42, 1494-1496.

Groom, C. R., Bruno, I. J., Lightfoot, M. P. \& Ward, S. C. (2016). Acta Cryst. B72, 171-179.

Hussain, S., Khan, I. U., Harrison, W. T. A. \& Tahir, M. N. (2015). J. Struct. Chem. 56, 934-941.

Kim, H.-C., Huh, S., Kim, J. Y., Moon, H. R., Lee, D. N. \& Kim, Y. (2017). CrystEngComm, 19, 99-109.

Legendziewicz, J., Keller, B., Turowska-Tyrk, I. \& Wojciechowski, W. (1999). New J. Chem. 23, 1097-1103.

Marsh, R. E. (2005). Acta Cryst. B61, 359.

Pagis, C., Ferbinteanu, M., Rothenberg, G. \& Tanase, S. (2016). ACS Catal. 6, 6063-6072.

Sheldrick, G. M. (2015a). Acta Cryst. A71, 3-8.

Sheldrick, G. M. (2015b). Acta Cryst. C71, 3-8. 


\section{supporting information}

Acta Cryst. (2022). E78, 159-163 [https://doi.org/10.1107/S2056989022000159]

\section{Crystal structure of a three-dimensional neodymium(III) coordination polymer, $\left[\mathrm{Nd}_{2}\left(\mathrm{H}_{2} \mathrm{O}\right)_{6} \text { (glutarato) }\left(\mathrm{SO}_{4}\right)_{2}\right]_{n}$}

\section{Saranphong Yimklan, Yothin Chimupala, Sutsiri Wongngam and Nippich Kaeosamut}

Computing details

Data collection: CrysAlis PRO (Agilent, 2014); cell refinement: CrysAlis PRO (Agilent, 2014); data reduction: CrysAlis PRO (Agilent, 2014); program(s) used to solve structure: SHELXT2018/2 (Sheldrick, 2015a); program(s) used to refine structure: SHELXL2018/3 (Sheldrick, 2015b); molecular graphics: OLEX2 (Dolomanov et al., 2009); software used to prepare material for publication: OLEX2 (Dolomanov et al., 2009).

Poly[hexaaqua $\left(\mu_{4}\right.$-glutarato)bis( $\mu_{3}$-sulfato)dineodymium(III)]

\section{Crystal data}

$\left[\mathrm{Nd}_{2}\left(\mathrm{C}_{5} \mathrm{H}_{6} \mathrm{O}_{4}\right)\left(\mathrm{SO}_{4}\right)_{2}\left(\mathrm{H}_{2} \mathrm{O}\right)_{6}\right]$

$M_{r}=718.79$

Monoclinic, $P 2{ }_{1} / c$

$a=15.5461(1) \AA$

$b=12.6621(1) \AA$

$c=8.8883(1) \AA$

$\beta=95.287(1)^{\circ}$

$V=1742.19(3) \AA^{3}$

$Z=4$

\section{Data collection}

SuperNova, Single source at offset/far, HyPix 3000 diffractometer

Radiation source: micro-focus sealed X-ray tube, SuperNova (Mo) X-ray Source

Mirror monochromator

$\omega$ scans

Absorption correction: multi-scan

(CrysAlisPro; Agilent, 2014)

Refinement

Refinement on $F^{2}$

Least-squares matrix: full

$R\left[F^{2}>2 \sigma\left(F^{2}\right)\right]=0.022$

$w R\left(F^{2}\right)=0.045$

$S=1.08$

3830 reflections

268 parameters

9 restraints
$F(000)=1376$

$D_{\mathrm{x}}=2.740 \mathrm{Mg} \mathrm{m}^{-3}$

Mo $K \alpha$ radiation, $\lambda=0.71073 \AA$

Cell parameters from 25026 reflections

$\theta=2.1-27.3^{\circ}$

$\mu=6.23 \mathrm{~mm}^{-1}$

$T=293 \mathrm{~K}$

Block, clear light violet

$0.2 \times 0.2 \times 0.2 \mathrm{~mm}$

$T_{\min }=0.448, T_{\max }=1.000$

38711 measured reflections

3830 independent reflections

3482 reflections with $I>2 \sigma(I)$

$R_{\text {int }}=0.067$

$\theta_{\text {max }}=27.4^{\circ}, \theta_{\min }=2.1^{\circ}$

$h=-20 \rightarrow 19$

$k=-16 \rightarrow 16$

$l=-11 \rightarrow 11$

Primary atom site location: structure-invariant direct methods

Secondary atom site location: difference Fourier map

Hydrogen site location: mixed

$\mathrm{H}$ atoms treated by a mixture of independent and constrained refinement

$w=1 /\left[\sigma^{2}\left(F_{\mathrm{o}}^{2}\right)+(0.0106 P)^{2}+1.2155 P\right]$

where $P=\left(F_{\mathrm{o}}^{2}+2 F_{\mathrm{c}}^{2}\right) / 3$ 
$(\Delta / \sigma)_{\max }=0.003$

$\Delta \rho_{\max }=0.62 \mathrm{e} \AA^{-3}$

$\Delta \rho_{\min }=-0.88$ e $\AA^{-3}$
Extinction correction: SHELXL2018/3

(Sheldrick 2015b),

$\mathrm{Fc}^{*}=\mathrm{kFc}\left[1+0.001 \mathrm{xFc}^{2} \lambda^{3} / \sin (2 \theta)\right]^{-1 / 4}$

Extinction coefficient: $0.00033(5)$

\section{Special details}

Geometry. All esds (except the esd in the dihedral angle between two 1.s. planes) are estimated using the full covariance matrix. The cell esds are taken into account individually in the estimation of esds in distances, angles and torsion angles; correlations between esds in cell parameters are only used when they are defined by crystal symmetry. An approximate (isotropic) treatment of cell esds is used for estimating esds involving l.s. planes.

Refinement. The structure of 1 was solved in the space group P21/c (No. 14) using direct methods in the SHELXT (Sheldrick, 2015a) structure-solution program and refined by full-matrix least-squares minimization on F2 using SHELXL 2018/3 (Sheldrick, 2015b).

Fractional atomic coordinates and isotropic or equivalent isotropic displacement parameters $\left(\AA^{2}\right)$

\begin{tabular}{|c|c|c|c|c|}
\hline & $x$ & $y$ & $z$ & $U_{\text {iso }} * / U_{\text {eq }}$ \\
\hline $\mathrm{Nd} 2$ & $1.07588(2)$ & 0.09859 (2) & $-0.12226(2)$ & $0.01075(6)$ \\
\hline $\mathrm{Nd} 1$ & $0.42661(2)$ & $0.43378(2)$ & $-0.18797(2)$ & $0.01254(6)$ \\
\hline $\mathrm{S} 2$ & $1.05205(6)$ & $0.38265(6)$ & $-0.21038(9)$ & $0.01194(18)$ \\
\hline $\mathrm{S} 1$ & $0.41004(6)$ & $0.35878(6)$ & $0.21539(9)$ & 0.01539 (19) \\
\hline O11 & $0.93262(15)$ & $0.07269(16)$ & $-0.0510(2)$ & $0.0157(5)$ \\
\hline O6 & $1.11665(15)$ & $0.45391(16)$ & $-0.1313(2)$ & $0.0180(5)$ \\
\hline $\mathrm{O} 5$ & $1.04359(16)$ & $0.28751(16)$ & $-0.1153(2)$ & $0.0177(5)$ \\
\hline O8 & $0.96805(15)$ & $0.43635(16)$ & $-0.2326(2)$ & $0.0155(5)$ \\
\hline O16W & $1.16897(17)$ & $0.0982(2)$ & $-0.3338(3)$ & $0.0265(6)$ \\
\hline H16A & 1.222796 & 0.084873 & -0.321649 & $0.040 *$ \\
\hline H16B & 1.152057 & 0.070890 & -0.418758 & $0.040^{*}$ \\
\hline O17W & $1.21843(18)$ & $0.1988(2)$ & $-0.0366(3)$ & $0.0321(7)$ \\
\hline H17A & $1.218(3)$ & $0.2626(15)$ & $-0.044(5)$ & $0.048 *$ \\
\hline H17B & $1.259(2)$ & $0.177(3)$ & $-0.083(4)$ & $0.048 *$ \\
\hline $\mathrm{O} 12$ & $0.80870(15)$ & $0.03120(18)$ & 0.0300 & $0.0220(6)$ \\
\hline O12W & $0.27085(16)$ & $0.37303(19)$ & $-0.2316(3)$ & $0.0258(6)$ \\
\hline H12A & 0.237228 & 0.422979 & -0.210018 & $0.039 *$ \\
\hline $\mathrm{H} 12 \mathrm{~B}$ & 0.258297 & 0.362463 & -0.325853 & $0.039 *$ \\
\hline O9 & $0.56170(17)$ & $0.41489(18)$ & $-0.0404(3)$ & $0.0236(6)$ \\
\hline $\mathrm{O} 2$ & $0.48805(16)$ & $0.42067(18)$ & $0.2617(3)$ & $0.0247(6)$ \\
\hline O13W & $0.36280(16)$ & $0.5016(2)$ & $-0.4426(3)$ & $0.0283(6)$ \\
\hline H13A & 0.309153 & 0.513771 & -0.439262 & $0.042 *$ \\
\hline H13B & 0.364813 & 0.453202 & -0.508542 & $0.042 *$ \\
\hline $\mathrm{O} 10$ & $0.67742(18)$ & $0.4309(2)$ & 0.1157 (3) & $0.0335(7)$ \\
\hline O14W & $0.5138(2)$ & $0.3668(2)$ & -0.4039 & $0.0425(8)$ \\
\hline H14A & $0.545(3)$ & $0.400(3)$ & $-0.463(4)$ & $0.064 *$ \\
\hline H14B & $0.498(3)$ & $0.313(2)$ & $-0.451(5)$ & $0.064^{*}$ \\
\hline $\mathrm{O} 1$ & $0.39367(18)$ & $0.3531(2)$ & $0.0516(3)$ & $0.0348(7)$ \\
\hline $\mathrm{C} 4$ & $0.8121(2)$ & $0.1900(3)$ & -0.1158 (4) & $0.0213(8)$ \\
\hline $\mathrm{H} 4 \mathrm{~A}$ & 0.776496 & 0.167837 & -0.205583 & $0.026^{*}$ \\
\hline H4B & 0.856900 & 0.236004 & -0.147741 & $0.026^{*}$ \\
\hline $\mathrm{C} 1$ & $0.6402(2)$ & $0.3905(3)$ & $-0.0018(4)$ & $0.0178(8)$ \\
\hline
\end{tabular}




\begin{tabular}{lllll} 
C5 & $0.8539(2)$ & $0.0942(2)$ & $-0.0408(4)$ & $0.0148(8)$ \\
C2 & $0.6882(3)$ & $0.3205(3)$ & $-0.1001(4)$ & $0.0297(10)$ \\
H2A & 0.715651 & 0.364043 & -0.171662 & $0.036^{*}$ \\
H2B & 0.647082 & 0.274906 & -0.157345 & $0.036^{*}$ \\
C3 & $0.7565(2)$ & $0.2524(3)$ & $-0.0148(4)$ & $0.0249(9)$ \\
H3A & 0.728270 & 0.203229 & 0.048365 & $0.030^{*}$ \\
H3B & 0.793796 & 0.297267 & 0.051188 & $0.030^{*}$ \\
O18 & $0.97596(18)$ & $0.14621(19)$ & $-0.3490(3)$ & $0.0206(6)$ \\
H18A & $0.945(2)$ & $0.095(2)$ & $-0.376(4)$ & $0.031^{*}$ \\
H18B & $0.992(2)$ & $0.172(3)$ & $-0.425(3)$ & $0.031^{*}$ \\
O7 & $1.08197(15)$ & $0.34921(17)$ & $-0.3553(2)$ & $0.0160(5)$ \\
O4 & $0.4228(2)$ & $0.2533(2)$ & $0.2767(3)$ & $0.0447(8)$ \\
O3 & $0.33686(18)$ & $0.4071(2)$ & $0.2790(3)$ & $0.0431(8)$ \\
\hline
\end{tabular}

Atomic displacement parameters $\left(\AA^{2}\right)$

\begin{tabular}{|c|c|c|c|c|c|c|}
\hline & $U^{11}$ & $U^{22}$ & $U^{33}$ & $U^{12}$ & $U^{13}$ & $U^{23}$ \\
\hline $\mathrm{Nd} 2$ & $0.01192(11)$ & $0.01076(10)$ & $0.00957(10)$ & $0.00054(7)$ & $0.00104(8)$ & $0.00014(7)$ \\
\hline $\mathrm{Nd} 1$ & $0.01289(12)$ & $0.01314(11)$ & $0.01148(10)$ & 0.00009 (7) & $0.00057(8)$ & $0.00022(7)$ \\
\hline $\mathrm{S} 2$ & $0.0158(5)$ & $0.0112(4)$ & 0.0089 (4) & $0.0013(3)$ & $0.0013(3)$ & 0.0007 (3) \\
\hline S1 & $0.0187(5)$ & $0.0147(4)$ & $0.0133(4)$ & $-0.0029(4)$ & 0.0047 (4) & $0.0003(3)$ \\
\hline O11 & $0.0119(13)$ & $0.0187(12)$ & $0.0161(13)$ & $0.0037(10)$ & $0.0000(11)$ & $-0.0004(10)$ \\
\hline O6 & $0.0206(14)$ & $0.0169(12)$ & $0.0154(12)$ & $-0.0016(11)$ & $-0.0037(11)$ & $-0.0019(10)$ \\
\hline O5 & $0.0272(15)$ & $0.0134(12)$ & $0.0128(12)$ & $0.0019(11)$ & $0.0028(11)$ & $0.0042(9)$ \\
\hline O8 & $0.0177(14)$ & $0.0140(12)$ & $0.0152(12)$ & $0.0039(10)$ & $0.0036(11)$ & $0.0022(9)$ \\
\hline O16W & $0.0175(15)$ & $0.0443(17)$ & $0.0177(14)$ & $-0.0005(13)$ & $0.0022(12)$ & $-0.0048(12)$ \\
\hline O17W & $0.0298(18)$ & $0.0261(15)$ & $0.0389(18)$ & $-0.0074(14)$ & $-0.0057(14)$ & $0.0082(14)$ \\
\hline O12 & $0.0154(14)$ & $0.0191(13)$ & $0.0313(14)$ & $0.0002(11)$ & $0.0010(12)$ & $0.0079(11)$ \\
\hline $\mathrm{O} 12 \mathrm{~W}$ & $0.0194(15)$ & $0.0312(15)$ & $0.0261(14)$ & $-0.0007(12)$ & $-0.0024(12)$ & $-0.0030(12)$ \\
\hline O9 & $0.0178(15)$ & $0.0285(14)$ & $0.0241(14)$ & $0.0071(11)$ & $0.0006(12)$ & $0.0010(11)$ \\
\hline $\mathrm{O} 2$ & $0.0240(16)$ & $0.0300(14)$ & $0.0193(14)$ & $-0.0128(12)$ & $-0.0021(12)$ & $0.0040(11)$ \\
\hline O13W & $0.0205(15)$ & $0.0439(17)$ & $0.0201(13)$ & $0.0049(13)$ & $0.0003(12)$ & $-0.0037(12)$ \\
\hline $\mathrm{O} 10$ & $0.0281(17)$ & $0.0360(16)$ & $0.0342(16)$ & $0.0110(13)$ & $-0.0090(14)$ & $-0.0181(13)$ \\
\hline O14W & $0.061(2)$ & $0.0426(19)$ & $0.0262(17)$ & $0.0041(17)$ & 0.0190 (16) & $-0.0004(14)$ \\
\hline $\mathrm{O} 1$ & $0.0471(19)$ & $0.0486(17)$ & $0.0084(12)$ & $-0.0274(15)$ & $0.0015(12)$ & $-0.0004(12)$ \\
\hline $\mathrm{C} 4$ & $0.020(2)$ & 0.0195 (19) & $0.024(2)$ & $0.0058(16)$ & $0.0006(16)$ & $0.0038(15)$ \\
\hline $\mathrm{C} 1$ & $0.015(2)$ & $0.0190(18)$ & $0.0188(19)$ & $0.0056(15)$ & $-0.0017(16)$ & $-0.0020(15)$ \\
\hline $\mathrm{C} 5$ & $0.015(2)$ & 0.0144 (17) & $0.0149(18)$ & $0.0013(15)$ & $-0.0009(15)$ & $-0.0031(14)$ \\
\hline $\mathrm{C} 2$ & $0.029(2)$ & $0.038(2)$ & $0.022(2)$ & 0.0139 (19) & $-0.0004(18)$ & $-0.0049(17)$ \\
\hline $\mathrm{C} 3$ & $0.030(2)$ & $0.023(2)$ & $0.0209(19)$ & $0.0139(17)$ & $0.0010(17)$ & $-0.0023(16)$ \\
\hline $\mathrm{O} 18$ & $0.0267(16)$ & $0.0193(14)$ & $0.0155(13)$ & $-0.0062(11)$ & $-0.0003(12)$ & $0.0043(11)$ \\
\hline $\mathrm{O} 7$ & $0.0188(14)$ & $0.0185(12)$ & $0.0111(11)$ & $0.0050(10)$ & $0.0030(10)$ & $-0.0002(9)$ \\
\hline $\mathrm{O} 4$ & $0.057(2)$ & $0.0187(15)$ & $0.0566(19)$ & $-0.0073(14)$ & $-0.0032(17)$ & $0.0138(14)$ \\
\hline $\mathrm{O} 3$ & $0.0171(16)$ & $0.069(2)$ & $0.0430(18)$ & $0.0099(14)$ & $0.0029(14)$ & $-0.0298(15)$ \\
\hline
\end{tabular}


Geometric parameters $\left(\AA,{ }^{\circ}\right)$

\begin{tabular}{|c|c|c|c|}
\hline $\mathrm{Nd} 2-\mathrm{O} 11$ & $2.393(2)$ & $\mathrm{O} 11-\mathrm{C} 5$ & $1.265(4)$ \\
\hline $\mathrm{Nd} 2-\mathrm{O} 11^{\mathrm{i}}$ & $2.670(2)$ & $\mathrm{O} 16 \mathrm{~W}-\mathrm{H} 16 \mathrm{~A}$ & 0.8508 \\
\hline $\mathrm{Nd} 2-\mathrm{O} 5$ & $2.446(2)$ & $\mathrm{O} 16 \mathrm{~W}-\mathrm{H} 16 \mathrm{~B}$ & 0.8501 \\
\hline $\mathrm{Nd} 2-\mathrm{O} 8^{\mathrm{ii}}$ & $2.487(2)$ & $\mathrm{O} 17 \mathrm{~W}-\mathrm{H} 17 \mathrm{~A}$ & $0.811(18)$ \\
\hline $\mathrm{Nd} 2-\mathrm{O} 16 \mathrm{~W}$ & $2.476(3)$ & $\mathrm{O} 17 \mathrm{~W}-\mathrm{H} 17 \mathrm{~B}$ & $0.836(18)$ \\
\hline $\mathrm{Nd} 2-\mathrm{O} 17 \mathrm{~W}$ & $2.606(3)$ & $\mathrm{O} 12-\mathrm{C} 5$ & $1.268(4)$ \\
\hline $\mathrm{Nd} 2-\mathrm{O} 12^{\mathrm{i}}$ & $2.514(2)$ & $\mathrm{O} 12 \mathrm{~W}-\mathrm{H} 12 \mathrm{~A}$ & 0.8534 \\
\hline $\mathrm{Nd} 2-\mathrm{O} 18$ & $2.502(2)$ & $\mathrm{O} 12 \mathrm{~W}-\mathrm{H} 12 \mathrm{~B}$ & 0.8534 \\
\hline $\mathrm{Nd} 2-\mathrm{O} 7^{\mathrm{iii}}$ & $2.456(2)$ & $\mathrm{O} 9-\mathrm{C} 1$ & $1.275(4)$ \\
\hline $\mathrm{Nd} 1-\mathrm{O} 12 \mathrm{~W}$ & $2.536(2)$ & O13W-H13A & 0.8513 \\
\hline $\mathrm{Nd} 1-\mathrm{O} 9^{\mathrm{iv}}$ & $2.785(2)$ & $\mathrm{O} 13 \mathrm{~W}-\mathrm{H} 13 \mathrm{~B}$ & 0.8508 \\
\hline $\mathrm{Nd} 1-\mathrm{O} 9$ & $2.383(2)$ & $\mathrm{O} 10-\mathrm{C} 1$ & $1.255(4)$ \\
\hline $\mathrm{Nd} 1-\mathrm{O} 2^{\mathrm{iv}}$ & $2.397(2)$ & $\mathrm{O} 14 \mathrm{~W}-\mathrm{H} 14 \mathrm{~A}$ & $0.854(19)$ \\
\hline $\mathrm{Nd} 1-\mathrm{O} 13 \mathrm{~W}$ & $2.536(2)$ & O14W-H14B & $0.831(18)$ \\
\hline $\mathrm{Nd} 1-\mathrm{O} 10^{\mathrm{iv}}$ & $2.481(3)$ & $\mathrm{C} 4-\mathrm{H} 4 \mathrm{~A}$ & 0.9700 \\
\hline $\mathrm{Nd} 1-\mathrm{O} 14 \mathrm{~W}$ & $2.592(3)$ & $\mathrm{C} 4-\mathrm{H} 4 \mathrm{~B}$ & 0.9700 \\
\hline $\mathrm{Nd} 1-\mathrm{O} 1$ & $2.457(2)$ & $\mathrm{C} 4-\mathrm{C} 5$ & $1.502(4)$ \\
\hline $\mathrm{Nd} 1-\mathrm{O}_{4}^{\mathrm{v}}$ & $2.390(2)$ & $\mathrm{C} 4-\mathrm{C} 3$ & $1.523(5)$ \\
\hline $\mathrm{S} 2-\mathrm{O} 6$ & $1.479(2)$ & $\mathrm{C} 1-\mathrm{C} 2$ & $1.491(5)$ \\
\hline $\mathrm{S} 2-\mathrm{O} 5$ & $1.485(2)$ & $\mathrm{C} 2-\mathrm{H} 2 \mathrm{~A}$ & 0.9700 \\
\hline $\mathrm{S} 2-\mathrm{O} 8$ & $1.470(2)$ & $\mathrm{C} 2-\mathrm{H} 2 \mathrm{~B}$ & 0.9700 \\
\hline $\mathrm{S} 2-\mathrm{O} 7$ & $1.472(2)$ & $\mathrm{C} 2-\mathrm{C} 3$ & $1.516(5)$ \\
\hline $\mathrm{S} 1-\mathrm{O} 2$ & $1.471(2)$ & $\mathrm{C} 3-\mathrm{H} 3 \mathrm{~A}$ & 0.9700 \\
\hline $\mathrm{S} 1-\mathrm{O} 1$ & $1.457(2)$ & C3-H3B & 0.9700 \\
\hline $\mathrm{S} 1-\mathrm{O} 4$ & $1.449(3)$ & $\mathrm{O} 18-\mathrm{H} 18 \mathrm{~A}$ & $0.827(18)$ \\
\hline $\mathrm{S} 1-\mathrm{O} 3$ & $1.452(3)$ & $\mathrm{O} 18-\mathrm{H} 18 \mathrm{~B}$ & $0.814(18)$ \\
\hline $\mathrm{O} 11-\mathrm{Nd} 2-\mathrm{O} 11^{\mathrm{i}}$ & $68.86(8)$ & $\mathrm{O} 8-\mathrm{S} 2-\mathrm{O} 6$ & $109.74(13)$ \\
\hline $\mathrm{O} 11-\mathrm{Nd} 2-\mathrm{O} 5$ & $85.94(8)$ & $\mathrm{O} 8-\mathrm{S} 2-\mathrm{O} 5$ & $109.14(14)$ \\
\hline $\mathrm{O} 11-\mathrm{Nd} 2-\mathrm{O}^{\mathrm{ii}}$ & $78.84(7)$ & $\mathrm{O} 8-\mathrm{S} 2-\mathrm{O} 7$ & $111.34(13)$ \\
\hline $\mathrm{O} 11-\mathrm{Nd} 2-\mathrm{O} 16 \mathrm{~W}$ & $145.48(8)$ & $\mathrm{O} 7-\mathrm{S} 2-\mathrm{O} 6$ & $109.63(14)$ \\
\hline $\mathrm{O} 11-\mathrm{Nd} 2-\mathrm{O} 17 \mathrm{~W}$ & $140.93(8)$ & $\mathrm{O} 7-\mathrm{S} 2-\mathrm{O} 5$ & $108.47(13)$ \\
\hline $\mathrm{O} 11-\mathrm{Nd} 2-\mathrm{O} 12^{\mathrm{i}}$ & $118.53(7)$ & $\mathrm{O} 1-\mathrm{S} 1-\mathrm{O} 2$ & $111.67(15)$ \\
\hline $\mathrm{O} 11-\mathrm{Nd} 2-\mathrm{O} 18$ & $73.89(8)$ & $\mathrm{O} 4-\mathrm{S} 1-\mathrm{O} 2$ & $107.78(16)$ \\
\hline $\mathrm{O} 11-\mathrm{Nd} 2-\mathrm{O} 7^{\mathrm{iii}}$ & $74.65(7)$ & $\mathrm{O} 4-\mathrm{S} 1-\mathrm{O} 1$ & $109.63(16)$ \\
\hline $\mathrm{O} 5-\mathrm{Nd} 2-\mathrm{O} 11^{\mathrm{i}}$ & $139.33(7)$ & $\mathrm{O} 4-\mathrm{S} 1-\mathrm{O} 3$ & $109.07(19)$ \\
\hline $\mathrm{O} 5-\mathrm{Nd} 2-\mathrm{O}^{\mathrm{ii}}$ & $140.68(7)$ & $\mathrm{O} 3-\mathrm{S} 1-\mathrm{O} 2$ & $108.79(16)$ \\
\hline $\mathrm{O} 5-\mathrm{Nd} 2-\mathrm{O} 16 \mathrm{~W}$ & $99.03(8)$ & $\mathrm{O} 3-\mathrm{S} 1-\mathrm{O} 1$ & $109.83(17)$ \\
\hline $\mathrm{O} 5-\mathrm{Nd} 2-\mathrm{O} 17 \mathrm{~W}$ & $71.78(8)$ & $\mathrm{Nd} 2-\mathrm{O} 11-\mathrm{Nd} 2^{\mathrm{i}}$ & $111.14(8)$ \\
\hline $\mathrm{O} 5-\mathrm{Nd} 2-\mathrm{O} 12^{\mathrm{i}}$ & $140.61(7)$ & $\mathrm{C} 5-\mathrm{O} 11-\mathrm{Nd} 2$ & $156.8(2)$ \\
\hline $\mathrm{O} 5-\mathrm{Nd} 2-\mathrm{O} 18$ & $70.81(7)$ & $\mathrm{C} 5-\mathrm{O} 11-\mathrm{Nd} 2^{\mathrm{i}}$ & $91.97(19)$ \\
\hline $\mathrm{O} 5-\mathrm{Nd} 2-\mathrm{O} 7^{\mathrm{iii}}$ & $72.67(7)$ & $\mathrm{S} 2-\mathrm{O} 5-\mathrm{Nd} 2$ & $138.58(13)$ \\
\hline $\mathrm{O} 8^{\mathrm{ii}}-\mathrm{Nd} 2-\mathrm{O} 11^{\mathrm{i}}$ & $66.58(7)$ & $\mathrm{S} 2-\mathrm{O} 8-\mathrm{Nd} 2^{\mathrm{vi}}$ & $130.54(13)$ \\
\hline $\mathrm{O} 8{ }^{\mathrm{ii}}-\mathrm{Nd} 2-\mathrm{O} 17 \mathrm{~W}$ & $137.65(9)$ & $\mathrm{Nd} 2-\mathrm{O} 16 \mathrm{~W}-\mathrm{H} 16 \mathrm{~A}$ & 122.8 \\
\hline $\mathrm{O} 8^{\mathrm{ii}}-\mathrm{Nd} 2-\mathrm{O} 12^{\mathrm{i}}$ & $77.46(7)$ & $\mathrm{Nd} 2-\mathrm{O} 16 \mathrm{~W}-\mathrm{H} 16 \mathrm{~B}$ & 121.4 \\
\hline $\mathrm{O} 8^{\mathrm{ii}}-\mathrm{Nd} 2-\mathrm{O} 18$ & $70.18(7)$ & $\mathrm{H} 16 \mathrm{~A}-\mathrm{O} 16 \mathrm{~W}-\mathrm{H} 16 \mathrm{~B}$ & 104.6 \\
\hline
\end{tabular}




\begin{tabular}{|c|c|c|c|}
\hline $\mathrm{O} 16 \mathrm{~W}-\mathrm{Nd} 2-\mathrm{O} 11^{\mathrm{i}}$ & $119.98(8)$ & $\mathrm{Nd} 2-\mathrm{O} 17 \mathrm{~W}-\mathrm{H} 17 \mathrm{~A}$ & $118(3)$ \\
\hline $\mathrm{O} 16 \mathrm{~W}-\mathrm{Nd} 2-\mathrm{O} 8^{\mathrm{ii}}$ & $75.90(8)$ & $\mathrm{Nd} 2-\mathrm{O} 17 \mathrm{~W}-\mathrm{H} 17 \mathrm{~B}$ & $111(3)$ \\
\hline $\mathrm{O} 16 \mathrm{~W}-\mathrm{Nd} 2-\mathrm{O} 17 \mathrm{~W}$ & $71.46(9)$ & $\mathrm{H} 17 \mathrm{~A}-\mathrm{O} 17 \mathrm{~W}-\mathrm{H} 17 \mathrm{~B}$ & $107(3)$ \\
\hline $\mathrm{O} 16 \mathrm{~W}-\mathrm{Nd} 2-\mathrm{O} 12^{\mathrm{i}}$ & $78.27(8)$ & $\mathrm{C} 5-\mathrm{O} 12-\mathrm{Nd} 2^{\mathrm{i}}$ & $99.3(2)$ \\
\hline $\mathrm{O} 16 \mathrm{~W}-\mathrm{Nd} 2-\mathrm{O} 18$ & $75.62(9)$ & $\mathrm{Nd} 1-\mathrm{O} 12 \mathrm{~W}-\mathrm{H} 12 \mathrm{~A}$ & 109.7 \\
\hline $\mathrm{O} 17 \mathrm{~W}-\mathrm{Nd} 2-\mathrm{O} 11^{\mathrm{i}}$ & $108.23(8)$ & $\mathrm{Nd} 1-\mathrm{O} 12 \mathrm{~W}-\mathrm{H} 12 \mathrm{~B}$ & 109.0 \\
\hline $\mathrm{O} 12^{\mathrm{i}}-\mathrm{Nd} 2-\mathrm{O} 11^{\mathrm{i}}$ & $49.67(7)$ & $\mathrm{H} 12 \mathrm{~A}-\mathrm{O} 12 \mathrm{~W}-\mathrm{H} 12 \mathrm{~B}$ & 104.3 \\
\hline $\mathrm{O} 12^{\mathrm{i}}-\mathrm{Nd} 2-\mathrm{O} 17 \mathrm{~W}$ & $70.17(8)$ & $\mathrm{Nd} 1-\mathrm{O} 9-\mathrm{Nd} 1^{\mathrm{iv}}$ & $109.09(9)$ \\
\hline $\mathrm{O} 18-\mathrm{Nd} 2-\mathrm{O} 11^{\mathrm{i}}$ & $126.89(7)$ & $\mathrm{C} 1-\mathrm{O} 9-\mathrm{Nd} 1^{\mathrm{iv}}$ & 88.51 (19) \\
\hline $\mathrm{O} 18-\mathrm{Nd} 2-\mathrm{O} 17 \mathrm{~W}$ & $124.49(8)$ & $\mathrm{C} 1-\mathrm{O} 9-\mathrm{Nd} 1$ & $161.0(2)$ \\
\hline $\mathrm{O} 18-\mathrm{Nd} 2-\mathrm{O} 12^{\mathrm{i}}$ & $142.32(8)$ & $\mathrm{S} 1-\mathrm{O} 2-\mathrm{Nd} 1^{\mathrm{iv}}$ & $142.78(14)$ \\
\hline 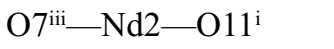 & $70.19(7)$ & $\mathrm{Nd} 1-\mathrm{O} 13 \mathrm{~W}-\mathrm{H} 13 \mathrm{~A}$ & 109.5 \\
\hline 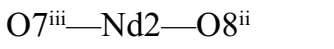 & $135.02(7)$ & $\mathrm{Nd} 1-\mathrm{O} 13 \mathrm{~W}-\mathrm{H} 13 \mathrm{~B}$ & 109.6 \\
\hline $\mathrm{O} 7 \mathrm{iii}-\mathrm{Nd} 2-\mathrm{O} 16 \mathrm{~W}$ & $139.53(8)$ & $\mathrm{H} 13 \mathrm{~A}-\mathrm{O} 13 \mathrm{~W}-\mathrm{H} 13 \mathrm{~B}$ & 104.6 \\
\hline $\mathrm{O} 7 \mathrm{iii}-\mathrm{Nd} 2-\mathrm{O} 17 \mathrm{~W}$ & $68.32(8)$ & $\mathrm{C} 1-\mathrm{O} 10-\mathrm{Nd} 1^{\mathrm{iv}}$ & $103.6(2)$ \\
\hline $\mathrm{O} 7^{\mathrm{iii}}-\mathrm{Nd} 2-\mathrm{O} 12^{\mathrm{i}}$ & $84.15(8)$ & $\mathrm{Nd} 1-\mathrm{O} 14 \mathrm{~W}-\mathrm{H} 14 \mathrm{~A}$ & $131(3)$ \\
\hline 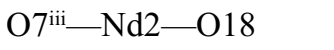 & $132.77(8)$ & $\mathrm{Nd} 1-\mathrm{O} 14 \mathrm{~W}-\mathrm{H} 14 \mathrm{~B}$ & $120(3)$ \\
\hline $\mathrm{O} 12 \mathrm{~W}-\mathrm{Nd} 1-\mathrm{O} 9^{\mathrm{iv}}$ & $108.50(8)$ & $\mathrm{H} 14 \mathrm{~A}-\mathrm{O} 14 \mathrm{~W}-\mathrm{H} 14 \mathrm{~B}$ & $104(3)$ \\
\hline $\mathrm{O} 12 \mathrm{~W}-\mathrm{Nd} 1-\mathrm{O} 14 \mathrm{~W}$ & $110.17(10)$ & $\mathrm{S} 1-\mathrm{O} 1-\mathrm{Nd} 1$ & $144.54(15)$ \\
\hline $\mathrm{O} 9-\mathrm{Nd} 1-\mathrm{O} 12 \mathrm{~W}$ & $146.52(8)$ & $\mathrm{H} 4 \mathrm{~A}-\mathrm{C} 4-\mathrm{H} 4 \mathrm{~B}$ & 107.7 \\
\hline $\mathrm{O} 9-\mathrm{Nd} 1-\mathrm{O} 9^{\mathrm{iv}}$ & $70.91(9)$ & $\mathrm{C} 5-\mathrm{C} 4-\mathrm{H} 4 \mathrm{~A}$ & 108.8 \\
\hline $\mathrm{O} 9-\mathrm{Nd} 1-\mathrm{O} 2^{\mathrm{iv}}$ & $75.27(8)$ & $\mathrm{C} 5-\mathrm{C} 4-\mathrm{H} 4 \mathrm{~B}$ & 108.8 \\
\hline $\mathrm{O} 9-\mathrm{Nd} 1-\mathrm{O} 13 \mathrm{~W}$ & $141.04(8)$ & $\mathrm{C} 5-\mathrm{C} 4-\mathrm{C} 3$ & $113.8(3)$ \\
\hline $\mathrm{O} 9-\mathrm{Nd} 1-\mathrm{O} 10^{\mathrm{iv}}$ & $119.29(8)$ & $\mathrm{C} 3-\mathrm{C} 4-\mathrm{H} 4 \mathrm{~A}$ & 108.8 \\
\hline $\mathrm{O} 9-\mathrm{Nd} 1-\mathrm{O} 14 \mathrm{~W}$ & $83.11(10)$ & $\mathrm{C} 3-\mathrm{C} 4-\mathrm{H} 4 \mathrm{~B}$ & 108.8 \\
\hline $\mathrm{O} 9-\mathrm{Nd} 1-\mathrm{O} 1$ & $74.03(9)$ & $\mathrm{O} 9-\mathrm{C} 1-\mathrm{Nd} 1^{\mathrm{iv}}$ & $66.63(18)$ \\
\hline $\mathrm{O} 9-\mathrm{Nd} 1-\mathrm{O} 4^{\mathrm{v}}$ & $89.01(9)$ & $\mathrm{O} 9-\mathrm{C} 1-\mathrm{C} 2$ & $120.3(3)$ \\
\hline $\mathrm{O} 2^{\mathrm{iv}}-\mathrm{Nd} 1-\mathrm{O} 12 \mathrm{~W}$ & $137.40(8)$ & $\mathrm{O} 10-\mathrm{C} 1-\mathrm{Nd} 1^{\mathrm{iv}}$ & 52.67 (17) \\
\hline $\mathrm{O} 2^{\mathrm{iv}}-\mathrm{Nd} 1-\mathrm{O} 9^{\mathrm{iv}}$ & $70.71(8)$ & $\mathrm{O} 10-\mathrm{C} 1-\mathrm{O} 9$ & $118.8(3)$ \\
\hline $\mathrm{O} 2^{\mathrm{iv}}-\mathrm{Nd} 1-\mathrm{O} 13 \mathrm{~W}$ & $71.12(8)$ & $\mathrm{O} 10-\mathrm{C} 1-\mathrm{C} 2$ & $120.8(3)$ \\
\hline $\mathrm{O} 2^{\mathrm{iv}}-\mathrm{Nd} 1-\mathrm{O} 10^{\mathrm{iv}}$ & $85.99(9)$ & $\mathrm{C} 2-\mathrm{C} 1-\mathrm{Nd} 1^{\mathrm{iv}}$ & $167.7(3)$ \\
\hline $\mathrm{O} 2^{\mathrm{iv}}-\mathrm{Nd} 1-\mathrm{O} 14 \mathrm{~W}$ & $73.06(9)$ & $\mathrm{O} 11-\mathrm{C} 5-\mathrm{Nd} 2^{\mathrm{i}}$ & $63.04(17)$ \\
\hline $\mathrm{O} 2^{\mathrm{iv}}-\mathrm{Nd} 1-\mathrm{O} 1$ & $136.14(8)$ & $\mathrm{O} 11-\mathrm{C} 5-\mathrm{O} 12$ & $118.9(3)$ \\
\hline $\mathrm{O} 13 \mathrm{~W}-\mathrm{Nd} 1-\mathrm{O} 12 \mathrm{~W}$ & $71.15(8)$ & $\mathrm{O} 11-\mathrm{C} 5-\mathrm{C} 4$ & $121.6(3)$ \\
\hline $\mathrm{O} 13 \mathrm{~W}-\mathrm{Nd} 1-\mathrm{O} 9^{\mathrm{iv}}$ & $114.29(7)$ & $\mathrm{O} 12-\mathrm{C} 5-\mathrm{Nd} 2^{\mathrm{i}}$ & $55.97(16)$ \\
\hline $\mathrm{O} 13 \mathrm{~W}-\mathrm{Nd} 1-\mathrm{O} 14 \mathrm{~W}$ & $68.79(9)$ & $\mathrm{O} 12-\mathrm{C} 5-\mathrm{C} 4$ & $119.5(3)$ \\
\hline $\mathrm{O} 10^{\mathrm{iv}}-\mathrm{Nd} 1-\mathrm{O} 12 \mathrm{~W}$ & $67.24(8)$ & $\mathrm{C} 4-\mathrm{C} 5-\mathrm{Nd} 2^{\mathrm{i}}$ & $175.3(3)$ \\
\hline $\mathrm{O} 10^{\mathrm{iv}}-\mathrm{Nd} 1-\mathrm{O} 9^{\mathrm{iv}}$ & $48.43(8)$ & $\mathrm{C} 1-\mathrm{C} 2-\mathrm{H} 2 \mathrm{~A}$ & 108.7 \\
\hline $\mathrm{O} 10^{\mathrm{iv}}-\mathrm{Nd} 1-\mathrm{O} 13 \mathrm{~W}$ & $77.67(9)$ & $\mathrm{C} 1-\mathrm{C} 2-\mathrm{H} 2 \mathrm{~B}$ & 108.7 \\
\hline $\mathrm{O} 10^{\mathrm{iv}}-\mathrm{Nd} 1-\mathrm{O} 14 \mathrm{~W}$ & $144.63(9)$ & $\mathrm{C} 1-\mathrm{C} 2-\mathrm{C} 3$ & $114.2(3)$ \\
\hline $\mathrm{O} 14 \mathrm{~W}-\mathrm{Nd} 1-\mathrm{O}^{\text {iv }}$ & $139.56(9)$ & $\mathrm{H} 2 \mathrm{~A}-\mathrm{C} 2-\mathrm{H} 2 \mathrm{~B}$ & 107.6 \\
\hline $\mathrm{O} 1-\mathrm{Nd} 1-\mathrm{O} 12 \mathrm{~W}$ & $74.58(8)$ & $\mathrm{C} 3-\mathrm{C} 2-\mathrm{H} 2 \mathrm{~A}$ & 108.7 \\
\hline $\mathrm{O} 1-\mathrm{Nd} 1-\mathrm{O}^{\mathrm{iv}}$ & $70.08(8)$ & $\mathrm{C} 3-\mathrm{C} 2-\mathrm{H} 2 \mathrm{~B}$ & 108.7 \\
\hline $\mathrm{O} 1-\mathrm{Nd} 1-\mathrm{O} 13 \mathrm{~W}$ & $144.93(9)$ & $\mathrm{C} 4-\mathrm{C} 3-\mathrm{H} 3 \mathrm{~A}$ & 108.7 \\
\hline $\mathrm{O} 1-\mathrm{Nd} 1-\mathrm{O} 10^{\mathrm{iv}}$ & $82.52(10)$ & $\mathrm{C} 4-\mathrm{C} 3-\mathrm{H} 3 \mathrm{~B}$ & 108.7 \\
\hline $\mathrm{O} 1-\mathrm{Nd} 1-\mathrm{O} 14 \mathrm{~W}$ & $132.11(10)$ & $\mathrm{C} 2-\mathrm{C} 3-\mathrm{C} 4$ & $114.2(3)$ \\
\hline $\mathrm{O} 4 \mathrm{v}-\mathrm{Nd} 1-\mathrm{O} 12 \mathrm{~W}$ & $70.57(9)$ & $\mathrm{C} 2-\mathrm{C} 3-\mathrm{H} 3 \mathrm{~A}$ & 108.7 \\
\hline $\mathrm{O} 4^{\mathrm{v}}-\mathrm{Nd} 1-\mathrm{O}^{9^{\mathrm{iv}}}$ & $140.98(9)$ & $\mathrm{C} 2-\mathrm{C} 3-\mathrm{H} 3 \mathrm{~B}$ & 108.7 \\
\hline
\end{tabular}




\begin{tabular}{|c|c|c|c|}
\hline $\mathrm{O} 4^{\mathrm{v}}-\mathrm{Nd} 1-\mathrm{O} 2^{\mathrm{iv}}$ & $137.22(10)$ & $\mathrm{H} 3 \mathrm{~A}-\mathrm{C} 3-\mathrm{H} 3 \mathrm{~B}$ & 107.6 \\
\hline $\mathrm{O} 4^{\mathrm{v}}-\mathrm{Nd} 1-\mathrm{O} 13 \mathrm{~W}$ & $102.45(9)$ & $\mathrm{Nd} 2-\mathrm{O} 18-\mathrm{H} 18 \mathrm{~A}$ & $110(3)$ \\
\hline $\mathrm{O} 4^{\mathrm{v}}-\mathrm{Nd} 1-\mathrm{O} 10^{\mathrm{iv}}$ & $135.22(10)$ & $\mathrm{Nd} 2-\mathrm{O} 18-\mathrm{H} 18 \mathrm{~B}$ & $123(3)$ \\
\hline $\mathrm{O} 4^{\mathrm{v}}-\mathrm{Nd} 1-\mathrm{O} 14 \mathrm{~W}$ & $65.60(10)$ & $\mathrm{H} 18 \mathrm{~A}-\mathrm{O} 18-\mathrm{H} 18 \mathrm{~B}$ & $107(3)$ \\
\hline $\mathrm{O} 4^{\mathrm{v}}-\mathrm{Nd} 1-\mathrm{O} 1$ & $72.38(10)$ & $\mathrm{S} 2-\mathrm{O} 7-\mathrm{Nd} 2^{\mathrm{v}}$ & $141.35(13)$ \\
\hline $\mathrm{O} 6-\mathrm{S} 2-\mathrm{O} 5$ & $108.45(13)$ & $\mathrm{S} 1-\mathrm{O} 4-\mathrm{Nd} 11^{\mathrm{iii}}$ & $164.56(18)$ \\
\hline $\mathrm{Nd} 2-\mathrm{O} 11-\mathrm{C} 5-\mathrm{Nd} 2^{\mathrm{i}}$ & $-174.9(5)$ & $\mathrm{O} 8-\mathrm{S} 2-\mathrm{O} 5-\mathrm{Nd} 2$ & $128.04(19)$ \\
\hline $\mathrm{Nd} 2-\mathrm{O} 11-\mathrm{C} 5-\mathrm{O} 12$ & $-178.5(3)$ & $\mathrm{O} 8-\mathrm{S} 2-\mathrm{O} 7-\mathrm{Nd} 2^{\mathrm{v}}$ & $32.0(3)$ \\
\hline $\mathrm{Nd} 2 \mathrm{i}-\mathrm{O} 11-\mathrm{C} 5-\mathrm{O} 12$ & $-3.5(3)$ & $\mathrm{O} 9-\mathrm{C} 1-\mathrm{C} 2-\mathrm{C} 3$ & $-148.9(3)$ \\
\hline $\mathrm{Nd} 2-\mathrm{O} 11-\mathrm{C} 5-\mathrm{C} 4$ & $4.0(7)$ & $\mathrm{O} 2-\mathrm{S} 1-\mathrm{O} 1-\mathrm{Nd} 1$ & $-18.1(4)$ \\
\hline $\mathrm{Nd} 2-\mathrm{O} 11-\mathrm{C} 5-\mathrm{C} 4$ & $179.0(3)$ & $\mathrm{O} 2-\mathrm{S} 1-\mathrm{O} 4-\mathrm{Nd} 1^{\mathrm{iii}}$ & $-137.7(7)$ \\
\hline $\mathrm{Nd} 2 \mathrm{i}-\mathrm{O} 12-\mathrm{C} 5-\mathrm{O} 11$ & $3.8(3)$ & $\mathrm{O} 10-\mathrm{C} 1-\mathrm{C} 2-\mathrm{C} 3$ & $34.9(5)$ \\
\hline $\mathrm{Nd} 2 \mathrm{i}-\mathrm{O} 12-\mathrm{C} 5-\mathrm{C} 4$ & $-178.6(2)$ & $\mathrm{O} 1-\mathrm{S} 1-\mathrm{O} 2-\mathrm{Nd} 1^{\mathrm{iv}}$ & $31.5(3)$ \\
\hline $\mathrm{Nd} 1-\mathrm{O} 9-\mathrm{C} 1-\mathrm{Nd} 1^{\mathrm{iv}}$ & $158.3(7)$ & $\mathrm{O} 1-\mathrm{S} 1-\mathrm{O} 4-\mathrm{Nd} 1^{\mathrm{iii}}$ & $-15.9(8)$ \\
\hline $\mathrm{Nd} 1-\mathrm{O} 9-\mathrm{C} 1-\mathrm{O} 10$ & $166.0(5)$ & $\mathrm{C} 1-\mathrm{C} 2-\mathrm{C} 3-\mathrm{C} 4$ & $-173.6(3)$ \\
\hline $\mathrm{Nd} 1^{\mathrm{iv}}-\mathrm{O} 9-\mathrm{C} 1-\mathrm{O} 10$ & $7.7(3)$ & $\mathrm{C} 5-\mathrm{C} 4-\mathrm{C} 3-\mathrm{C} 2$ & $-157.3(3)$ \\
\hline $\mathrm{Nd} 1^{\mathrm{iv}}-\mathrm{O} 9-\mathrm{C} 1-\mathrm{C} 2$ & $-168.6(3)$ & $\mathrm{C} 3-\mathrm{C} 4-\mathrm{C} 5-\mathrm{O} 11$ & $-134.7(3)$ \\
\hline $\mathrm{Nd} 1-\mathrm{O} 9-\mathrm{C} 1-\mathrm{C} 2$ & $-10.3(9)$ & $\mathrm{C} 3-\mathrm{C} 4-\mathrm{C} 5-\mathrm{O} 12$ & $47.8(4)$ \\
\hline $\mathrm{Nd} 1^{\mathrm{iv}}-\mathrm{O} 10-\mathrm{C} 1-\mathrm{O} 9$ & $-8.9(4)$ & $\mathrm{O} 7-\mathrm{S} 2-\mathrm{O} 5-\mathrm{Nd} 2$ & $6.6(2)$ \\
\hline $\mathrm{Nd} 1^{\mathrm{iv}}-\mathrm{O} 10-\mathrm{C} 1-\mathrm{C} 2$ & $167.4(3)$ & $\mathrm{O} 7-\mathrm{S} 2-\mathrm{O} 8-\mathrm{Nd} 2^{\mathrm{vi}}$ & $-59.51(19)$ \\
\hline $\mathrm{Nd} 1^{\mathrm{iv}}-\mathrm{C} 1-\mathrm{C} 2-\mathrm{C} 3$ & $89.6(11)$ & $\mathrm{O} 4-\mathrm{S} 1-\mathrm{O} 2-\mathrm{Nd} 1^{\mathrm{iv}}$ & $151.9(3)$ \\
\hline $\mathrm{O} 6-\mathrm{S} 2-\mathrm{O} 5-\mathrm{Nd} 2$ & $-112.4(2)$ & $\mathrm{O} 4-\mathrm{S} 1-\mathrm{O} 1-\mathrm{Nd} 1$ & $-137.5(3)$ \\
\hline $\mathrm{O} 6-\mathrm{S} 2-\mathrm{O} 8-\mathrm{Nd} 2^{\mathrm{vi}}$ & 62.05 (19) & $\mathrm{O} 3-\mathrm{S} 1-\mathrm{O} 2-\mathrm{Nd} 1^{\mathrm{iv}}$ & $-89.9(3)$ \\
\hline $\mathrm{O} 6-\mathrm{S} 2-\mathrm{O} 7-\mathrm{Nd} 2^{\mathrm{v}}$ & $-89.7(2)$ & $\mathrm{O} 3-\mathrm{S} 1-\mathrm{O} 1-\mathrm{Nd} 1$ & $102.7(3)$ \\
\hline $\mathrm{O} 5-\mathrm{S} 2-\mathrm{O} 8-\mathrm{Nd} 2^{\mathrm{vi}}$ & $-179.23(14)$ & $\mathrm{O} 3-\mathrm{S} 1-\mathrm{O} 4-\mathrm{Nd} 1^{\mathrm{iii}}$ & $104.4(8)$ \\
\hline $\mathrm{O} 5-\mathrm{S} 2-\mathrm{O} 7-\mathrm{Nd} 2^{\mathrm{v}}$ & $152.09(19)$ & & \\
\hline
\end{tabular}

Symmetry codes: (i) $-x+2,-y,-z$; (ii) $-x+2, y-1 / 2,-z-1 / 2$; (iii) $x,-y+1 / 2, z+1 / 2$; (iv) $-x+1,-y+1,-z$; (v) $x,-y+1 / 2, z-1 / 2$; (vi) $-x+2, y+1 / 2,-z-1 / 2$.

Hydrogen-bond geometry $\left(A,{ }^{\circ}\right)$

\begin{tabular}{|c|c|c|c|c|}
\hline$D-\mathrm{H} \cdots A$ & $D-\mathrm{H}$ & $\mathrm{H} \cdots A$ & $D \cdots A$ & $D-\mathrm{H} \cdots A$ \\
\hline $\mathrm{O} 16 W-\mathrm{H} 16 A \cdots \mathrm{O}^{\mathrm{vii}}$ & 0.85 & 1.91 & $2.709(4)$ & 155 \\
\hline $\mathrm{O} 16 W-\mathrm{H} 16 B^{\cdots} \mathrm{O}^{\mathrm{v}}$ & 0.85 & 1.94 & $2.774(3)$ & 165 \\
\hline $\mathrm{O} 17 W-\mathrm{H} 17 A \cdots \mathrm{O} 12 W^{\mathrm{viii}}$ & $0.81(2)$ & $2.38(4)$ & $2.966(4)$ & $130(4)$ \\
\hline $\mathrm{O} 17 W-\mathrm{H} 17 B \cdots \mathrm{O} 3^{\mathrm{vii}}$ & $0.84(2)$ & $2.09(2)$ & $2.903(4)$ & $165(4)$ \\
\hline $\mathrm{O} 12 W-\mathrm{H} 12 A \cdots \mathrm{O}^{\mathrm{ix}}$ & 0.85 & 2.10 & $2.825(4)$ & 143 \\
\hline $\mathrm{O} 12 W-\mathrm{H} 12 B \cdots \mathrm{O} 17 W^{\mathrm{x}}$ & 0.85 & 2.07 & $2.904(4)$ & 166 \\
\hline $\mathrm{O} 13 W-\mathrm{H} 13 A \cdots \mathrm{O} 12^{\mathrm{xi}}$ & 0.85 & 1.95 & $2.733(3)$ & 153 \\
\hline $\mathrm{O} 13 W-\mathrm{H} 13 B \cdots \mathrm{O} 3^{\mathrm{xii}}$ & 0.85 & 1.99 & $2.745(4)$ & 148 \\
\hline $\mathrm{O} 14 W-\mathrm{H} 14 A \cdots \mathrm{O} 13 W^{\mathrm{xiii}}$ & $0.85(2)$ & $2.13(2)$ & $2.966(4)$ & $165(4)$ \\
\hline $\mathrm{O} 18-\mathrm{H} 18 A \cdots \mathrm{O} 6^{\mathrm{ii}}$ & $0.83(2)$ & $2.03(2)$ & $2.826(3)$ & $160(3)$ \\
\hline $\mathrm{O} 18-\mathrm{H} 18 B^{\cdots \mathrm{O}^{\mathrm{v}}}$ & $0.81(2)$ & $2.00(2)$ & $2.805(3)$ & $170(4)$ \\
\hline
\end{tabular}

Symmetry codes: (ii) $-x+2, y-1 / 2,-z-1 / 2$; (v) $x,-y+1 / 2, z-1 / 2$; (vii) $x+1,-y+1 / 2, z-1 / 2$; (viii) $x+1, y, z$; (ix) $x-1, y, z$; (x) $x-1,-y+1 / 2, z-1 / 2$; (xi) $-x+1$, $y+1 / 2,-z-1 / 2$; (xii) $x, y, z-1$; (xiii) $-x+1,-y+1,-z-1$. 